\title{
Correction to: RC-Security Mechanism to Prevent Attacks on IoT Smart Switch
}

\author{
Jigar Makhija (i] and V. Anantha Narayanan (1)
}

\section{Correction to: \\ Chapter "RC-Security Mechanism to Prevent Attacks on IoT Smart Switch" in: N. Chaubey et al. (Eds.): Computing \\ Science, Communication and Security, CCIS 1416, https://doi.org/10.1007/978-3-030-76776-1_17}

In the originally published version of chapter 17, the name of the Author was incorrect. The Author's name has been corrected as "V. Anantha Narayanan". 